\title{
STRONG CONVERGENCE OF APPROXIMATION FIXED POINTS FOR NONEXPANSIVE NONSELF-MAPPING
}

\author{
RUDONG CHEN AND ZHICHUAN ZHU
}

Received 17 May 2006; Accepted 22 June 2006

Let $C$ be a closed convex subset of a uniformly smooth Banach space $E$, and $T: C \rightarrow E$ a nonexpansive nonself-mapping satisfying the weakly inwardness condition such that $F(T) \neq \varnothing$, and $f: C \rightarrow C$ a fixed contractive mapping. For $t \in(0,1)$, the implicit iterative sequence $\left\{x_{t}\right\}$ is defined by $x_{t}=P\left(t f\left(x_{t}\right)+(1-t) T x_{t}\right)$, the explicit iterative sequence $\left\{x_{n}\right\}$ is given by $x_{n+1}=P\left(\alpha_{n} f\left(x_{n}\right)+\left(1-\alpha_{n}\right) T x_{n}\right)$, where $\alpha_{n} \in(0,1)$ and $P$ is a sunny nonexpansive retraction of $E$ onto $C$. We prove that $\left\{x_{t}\right\}$ strongly converges to a fixed point of $T$ as $t \rightarrow 0$, and $\left\{x_{n}\right\}$ strongly converges to a fixed point of $T$ as $\alpha_{n}$ satisfying appropriate conditions. The results presented extend and improve the corresponding results of Hong-Kun Xu (2004) and Yisheng Song and Rudong Chen (2006).

Copyright (c) 2006 Hindawi Publishing Corporation. All rights reserved.

\section{Introduction}

Let $C$ be a nonempty closed convex subset of a Banach space $E$, and Let $T: C \rightarrow C$ be a nonexpansive mapping (i.e., $\|T x-T y\| \leq\|x-y\|$ for all $x, y \in C$ ). We use $\operatorname{Fix}(T)$ to denote the set of fixed points of $T$; that is , $\operatorname{Fix}(T)=\{x \in C: x=T x\}$. Recall that a selfmapping $f: C \rightarrow C$ is a contraction on $C$ if there exists a constant $\beta \in(0,1)$ such that

$$
\|f(x)-f(y)\| \leq \beta\|x-y\|, \quad x, y \in C .
$$

$\mathrm{Xu}$ (see [6]) defined the following two viscosity iterations for nonexpansive mappings:

$$
\begin{gathered}
x_{t}=t f\left(x_{t}\right)+(1-t) T x_{t}, \quad x \in C, \\
x_{n+1}=\alpha_{n} f\left(x_{n}\right)+\left(1-\alpha_{n}\right) T x_{n},
\end{gathered}
$$

where $\alpha_{n}$ is a sequence in $(0,1)$. Xu proved the strong convergence of $\left\{x_{t}\right\}$ defined by $(1.2)$ as $t \rightarrow 0$ and $\left\{x_{n}\right\}$ defined by (1.3) in both Hilbert space and uniformly smooth Banach space. 
Recently, Song and Chen [2] proved if $C$ is a closed subset of a real reflexive Banach space $E$ which admits a weakly sequentially continuous duality mapping from $E$ to $E^{*}$, and if $T: C \rightarrow E$ is a nonexpansive nonself-mapping satisfying the weakly inward condition, $F(T) \neq \phi, f: C \rightarrow C$ is a fixed contractive mapping, and $P$ is a sunny nonexpansive retraction of $E$ onto $C$, then the sequences $\left\{x_{t}\right\}$ and $\left\{x_{n}\right\}$ defined by

$$
\begin{gathered}
x_{t}=P\left(t f\left(x_{t}\right)+(1-t) T x_{t}\right), \\
x_{n+1}=P\left(\alpha_{n} f\left(x_{n}\right)+\left(1-\alpha_{n}\right) T x_{n}\right)
\end{gathered}
$$

strongly converge to a fixed point of $T$.

In this paper, we establish the strong convergence of both $\left\{x_{t}\right\}$ defined by (1.4) and $\left\{x_{n}\right\}$ defined by (1.5) for a nonexpansive nonself-mapping $T$ in a uniformly smooth Banach space. Our results extend and improve the results in $[2,6]$.

\section{Preliminaries}

Let $E$ be a real Banach space and let $J$ denote the normalized duality mapping from $E$ into $2^{E^{*}}$ given by

$$
J(x)=\left\{f \in E^{*}:\langle x, f\rangle=\|x\|\|f\|,\|x\|=\|f\|\right\} \quad \forall x \in E,
$$

where $E^{*}$ denotes the dual space of $E$ and $\langle\cdot, \cdot\rangle$ denotes the generalized duality pairing. In the sequence, we will denote the single-valued duality mapping by $j$, and $x_{n} \rightarrow x$ will denote strong convergence of the sequence $\left\{x_{n}\right\}$ to $x$. In Banach space $E$, the following result is well known $[1,3]$ for all $x, y \in E$, for all $j(x+y) \in J(x+y)$, for all $j(x) \in J(x)$,

$$
\|x\|^{2}+2\langle y, j(x)\rangle \leq\|x+y\|^{2} \leq\|x\|^{2}+2\langle y, j(x+y)\rangle .
$$

Recall that the norm of $E$ is said to be Gâteaux differentiable (and $E$ is said to be smooth) if

$$
\lim _{t \rightarrow 0} \frac{\|x+t y\|-\|x\|}{t}
$$

exists for each $x, y$ in its unit sphere $U=\{x \in E:\|x\|=1\}$. It is said to be uniformly Gâteaux differentiable if, for each $y \in U$, this limit is attained uniformly for $x \in U$. Finally, the norm is said to be uniformly Fréchet differentiable (and $E$ is said to be uniformly smooth) if the limit in (2.3) is attained uniformly for $(x, y) \in U \times U$. A Banach space $E$ is said to be smooth if and only if $J$ is single valued. It is also well known that if $E$ is uniformly smooth, $J$ is uniformly norm-to-norm continuous. These concepts may be found in $[3]$.

If $C$ and $D$ are nonempty subsets of a Banach space $E$ such that $C$ is nonempty closed convex and $D \subset C$, then a mapping $P: C \rightarrow D$ is called a retraction from $C$ to $D$ if $P^{2}=P$. It is easily known that a mapping $P: C \rightarrow D$ is retraction, then $P x=x$, for all $x \in D$. A mapping $P: C \rightarrow D$ is called sunny if

$$
P(P x+t(x-P x))=P x \quad \forall x \in C,
$$


whenever $P x+t(x-P x) \in C$ and $t>0$. A subset $D$ of $C$ is said to be a sunny nonexpansive retract of $C$ if there exists a sunny nonexpansive retraction of $C$ onto $D$. For more detail, see $[1,3-5]$.

The following lemma is well known [3].

LeMma 2.1. Let $C$ be a nonempty convex subset of a smooth Banach space $E, D \in C, J: E \rightarrow$ $E^{*}$ the (normalized) duality mapping of $E$, and $P: C \rightarrow D$ a retraction. Then the following are equivalent:

(i) $\langle x-P x, j(y-P x)\rangle \leq 0$ for all $x \in C$ and $y \in D$;

(ii) $P$ is both sunny and nonexpansive.

Let $C$ be a nonempty convex subset of a Banach space $E$, then for $x \in C$, we define the inward set $[4,5]$ :

$$
I_{C}(x)=\{y \in E: y=x+\lambda(z-x), z \in C \text { and } \lambda \geq 0\}
$$

A mapping $T: C \rightarrow E$ is said to be satisfying the inward condition if $T x \in I_{C}(x)$ for all $x \in C$. $T$ is also said to be satisfying the weakly inward condition if for each $x \in C, T x \in$ $\overline{I_{C}(x)}\left(\overline{I_{C}(x)}\right.$ is the closure of $\left.I_{C}(x)\right)$. Clearly $C \subset I_{C}(x)$ and it is not hard to show that $I_{C}(x)$ is a convex set as $C$ is. Using above these results and definitions, we can easily show the following lemma.

Lemma 2.2 ([2], Lemma 1.2). Let $C$ be a nonempty closed subset of a smooth Banach space $E$, let $T: C \rightarrow E$ be nonexpansive nonself-mapping satisfying the weakly inward condition, and let $P$ be a sunny nonexpansive retraction of $E$ onto $C$. Then $F(T)=F(P T)$.

Lemma 2.3 ([2], Lemma 2.1). Let $E$ be a Banach space and let $C$ be a nonempty closed convex subset of $E$. Suppose that $T: C \rightarrow E$ is a nonexpansive mapping such that for each fixed contractive mapping $f: C \rightarrow C$, and $P$ is a sunny nonexpansive retraction of $E$ onto $C$. For each $t \in(0,1),\left\{x_{t}\right\}$ is defined by (1.4). Suppose $u \in C$ is a fixed point of $T$, then

(i) $\left\langle x_{t}-f\left(x_{t}\right), j\left(x_{t}-u\right)\right\rangle \leq 0$;

(ii) $\left\{x_{t}\right\}$ is bounded.

Definition 2.4. $\mu$ is called a Banach limit if $\mu$ is a continuous linear functional on $l^{\infty}$ satisfying

(i) $\|\mu(e)\|=1=\mu(1), e=(1,1,1, \ldots)$;

(ii) $\mu_{n}\left(a_{n}\right)=\mu_{n}\left(a_{n+1}\right)$, for all $a_{n} \in\left(a_{0}, a_{1}, \ldots\right) \in l^{\infty}$;

(iii) $\liminf _{n \rightarrow \infty} a_{n} \leq \mu\left(a_{n}\right) \leq \limsup _{n \rightarrow \infty} a_{n}$, for all $a_{n} \in\left(a_{0}, a_{1}, \ldots\right) \in l^{\infty}$.

According to time and circumstances, we use $\mu_{n}\left(a_{n}\right)$ instead of $\mu\left(a_{0}, a_{1}, \ldots\right)$.

Further, we know the following result.

Lemma 2.5 ([3], Lemma 4.5.4). Let $C$ be a nonempty closed convex subset of a Banach space $E$ with a uniformly Gâteaux differentiable norm and let $\left\{x_{n}\right\}$ be a bounded sequence in $E$. Let $\mu$ be a Banach limit and $u \in C$. Then

$$
\mu_{n}\left\|x_{n}-u\right\|^{2}=\min _{y \in C} \mu_{n}\left\|x_{n}-y\right\|^{2}
$$


4 Strong convergence of approximation fixed points

if and only if

$$
\mu_{n}\left\langle x-u, J\left(x_{n}-u\right)\right\rangle \leq 0
$$

for all $x \in C$.

\section{Main results}

Theorem 3.1. Let $E$ be a uniformly smooth Banach, suppose that $C$ is a nonempty closed convex subset of $E$ and $T: C \rightarrow E$ is a nonexpansive nonself-mapping satisfying the weakly inward condition and $F(T) \neq \varnothing$. Let $f: C \rightarrow C$ be a fixed contractive mapping, and let $\left\{x_{t}\right\}$ be defined by (1.4), where $P$ is a sunny nonexpansive retraction of $E$ onto $C$. Then as $t \rightarrow 0$ $\left\{x_{t}\right\}$ converges strongly to some fixed point $q$ of $T$ that $q$ is the unique solution in $F(T)$ to the following variational inequality:

$$
\langle(I-f) q, j(q-u)\rangle \leq 0 \quad \forall u \in F(T) .
$$

Proof. For all $u \in F(T)$ by Lemma 2.3(ii), $\left\{x_{t}\right\}$ is bounded, therefore the sets $\left\{T x_{t}: t \in\right.$ $(0,1)\}$ and $\left\{f\left(x_{t}\right): t \in(0,1)\right\}$ are also bounded. From $x_{t}=P\left(t f\left(x_{t}\right)+(1-t) T x_{t}\right)$, we have

$$
\begin{aligned}
\left\|x_{t}-P T x_{t}\right\| & =\left\|P\left(t f\left(x_{t}\right)+(1-t) T x_{t}\right)-P T x_{t}\right\| \\
& \leq\left\|t f\left(x_{t}\right)+(1-t) T x_{t}-T x_{t}\right\| \\
& =t\left\|T x_{t}-f\left(x_{t}\right)\right\| \longrightarrow 0 \text { as } t \longrightarrow 0 .
\end{aligned}
$$

This implies that

$$
\lim _{t \rightarrow 0}\left\|x_{t}-P T x_{t}\right\|=0
$$

Assume $t_{n} \rightarrow 0$, set $x_{n}:=x_{t_{n}}$, and define $g: C \rightarrow \mathbb{R}$ by $g(x)=\mu_{n}\left\|x_{n}-x\right\|^{2}, x \in C$, where $\mu_{n}$ is a Banach limit on $\ell^{\infty}$. Let

$$
K=\left\{x \in C: g(x)=\min _{y \in C} \mu_{n}\left\|x_{n}-y\right\|^{2}\right\} .
$$

It is easily seen that $K$ is a nonempty closed convex bounded subset of $E$, since (note $\left.\left\|x_{n}-T x_{n}\right\| \rightarrow 0\right)$

$$
g(T x)=\mu_{n}\left\|x_{n}-T x\right\|^{2}=\mu_{n}\left\|T x_{n}-T x\right\|^{2} \leq \mu_{n}\left\|x_{n}-x\right\|^{2}=g(x) .
$$

It follows that $T(K) \subset K$, that is, $K$ is invariant under $T$. Since a uniformly smooth Banach space has the fixed point property for nonexpansive mappings, $T$ has a fixed point, say $q$, in $K$. From Lemma 2.5 we get

$$
\mu_{n}\left\langle x-q, j\left(x_{n}-q\right)\right\rangle \leq 0, \quad x \in C .
$$


For all $q \in F(T)$, we have $t f\left(x_{t}\right)+(1-t) q=P\left[t f\left(x_{t}\right)+(1-t) q\right]$, then

$$
\begin{aligned}
\| x_{t}- & {\left[t f\left(x_{t}\right)+(1-t) q\right] \| } \\
& =\left\|P\left[t f\left(x_{t}\right)+(1-t) T x_{t}\right]-P\left[t f\left(x_{t}\right)+(1-t) q\right]\right\| \\
& \leq\left\|(1-t)\left(T x_{t}-q\right)\right\| \leq(1-t)\left\|x_{t}-q\right\| .
\end{aligned}
$$

Hence from (2.2) and the above inequality we get

$$
\begin{aligned}
\| x_{t}- & {\left[t f\left(x_{t}\right)+(1-t) q\right] \|^{2} } \\
& =\left\|(1-t)\left(x_{t}-q\right)+t\left(x_{t}-f\left(x_{t}\right)\right)\right\|^{2} \\
& \geq(1-t)^{2}\left\|x_{t}-q\right\|^{2}+2 t(1-t)\left\langle x_{t}-f\left(x_{t}\right), j\left(x_{t}-q\right)\right\rangle .
\end{aligned}
$$

Therefore

$$
\left\langle x_{t}-f\left(x_{t}\right), j\left(x_{t}-q\right)\right\rangle \leq 0 \text {. }
$$

Then

$$
\begin{aligned}
0 & \geq\left\langle x_{t}-f\left(x_{t}\right), j\left(x_{t}-q\right)\right\rangle \\
& =\left\|x_{t}-q\right\|^{2}+\left\langle q-f(q), j\left(x_{t}-q\right)\right\rangle+\left\langle f(q)-f\left(x_{t}\right), j\left(x_{t}-q\right)\right\rangle \\
& \geq(1-\beta)\left\|x_{t}-q\right\|^{2}+\left\langle q-f(q), j\left(x_{t}-q\right)\right\rangle .
\end{aligned}
$$

We get

$$
\left\|x_{t}-q\right\|^{2} \leq \frac{1}{1-\beta}\left\langle f(q)-q, j\left(x_{t}-q\right)\right\rangle .
$$

Now applying Banach limit to the above inequality, we get

$$
\mu_{n}\left\|x_{t}-q\right\|^{2} \leq \mu_{n}\left(\frac{1}{1-\beta}\left\langle f(q)-q, j\left(x_{t}-q\right)\right\rangle\right) .
$$

Let $x=f(q)$ in (3.6), and noting (3.12), we have

$$
\mu_{n}|| x_{t}-q \|^{2} \leq 0
$$

that is,

$$
\mu_{n}\left\|x_{n}-q\right\|^{2}=0
$$

and then exists a subsequence which is still denoted by $\left\{x_{n}\right\}$ such that

$$
x_{n} \longrightarrow q, \quad n \longrightarrow \infty
$$

We have proved that for any sequence $\left\{x_{t_{n}}\right\}$ in $\left\{x_{t}: t \in(0,1)\right\}$, there exists a subsequence which is still denoted by $\left\{x_{t_{n}}\right\}$ that converges to some point $q$ of $T$. To prove that 
6 Strong convergence of approximation fixed points

the entire net $\left\{x_{t}\right\}$ converges to $q$, suppose that there exists another sequence $\left\{x_{s_{k}}\right\} \subset\left\{x_{t}\right\}$ such that $x_{s_{k}} \rightarrow p$, as $s_{k} \rightarrow 0$, then we also have $p \in F(T)$ (using $\lim _{t \rightarrow 0}\left\|x_{t}-P T x_{t}\right\|=0$ ). Next we show $p=q$ and $q$ is the unique solution in $F(T)$ to the following variational inequality:

$$
\langle(I-f) q, j(q-u)\rangle \quad \forall u \in F(T) .
$$

Since the sets $\left\{x_{t}-u\right\}$ and $\left\{x_{t}-f\left(x_{t}\right)\right\}$ are bounded and the uniform smoothness of $E$ implies that the duality map $J$ is norm-to-norm uniformly continuous on bounded sets of $E$, for any $u \in F(T)$, by $x_{s_{k}} \rightarrow p\left(s_{k} \rightarrow 0\right)$, we have

$$
\begin{gathered}
\left\|(I-f) x_{s_{k}}-(I-f) p\right\| \longrightarrow 0, \quad s_{k} \longrightarrow 0, \\
\left|\left\langle x_{s_{k}}-f\left(x_{s_{k}}\right), j\left(x_{s_{k}}-u\right)\right\rangle-\langle(I-f) p, j(p-u)\rangle\right| \\
=\left|\left\langle x_{s_{k}}-f\left(x_{s_{k}}\right)-(I-f) p, j\left(x_{s_{k}}-u\right)\right\rangle-\left\langle(I-f) p, j\left(x_{s_{k}}-u\right)-j(p-u)\right\rangle\right| \\
\leq||(I-f) x_{s_{k}}-(I-f) p\left\||| x_{s_{k}}-u\right\| \\
+\left|\left\langle(I-f) p, j\left(x_{s_{k}}-u\right)-j(p-u)\right\rangle\right| \longrightarrow 0 \quad \text { as } s_{k} \longrightarrow 0 .
\end{gathered}
$$

Therefore, noting Lemma 2.3(i), for any $u \in F(T)$, we get

$$
\langle(I-f) p, j(p-u)\rangle=\lim _{s_{k} \rightarrow 0}\left\langle x_{s_{k}}-f\left(x_{s_{k}}\right), j\left(x_{s_{k}}-u\right)\right\rangle \leq 0 .
$$

Similarly, we also can show

$$
\langle(I-f) q, j(q-u)\rangle=\left\langle x_{t_{n}}-f\left(x_{t_{n}}\right), j\left(x_{t_{n}}-u\right)\right\rangle \leq 0 .
$$

Interchange $q$ and $u$ to obtain

$$
\langle(I-f) p, j(p-q)\rangle \leq 0 .
$$

Interchange $p$ and $u$ to obtain

$$
\langle(I-f) q, j(q-p)\rangle \leq 0 .
$$

This implies that

$$
\langle(p-q)-(f(p)-f(q)), j(p-q)\rangle \leq 0,
$$

that is,

$$
\|p-q\|^{2} \leq \beta\|p-q\|^{2}
$$

This is a contradiction, so we must have $q=p$.

The proof is complete. 
From Theorem 3.1 we can get the following corollary directly.

Corollary 3.2. Let $E$ be a uniformly smooth space, suppose $C$ is a nonempty closed convex subset of $E, T: C \rightarrow E$ is a nonexpansive mapping satisfying the weakly inward condition, and $F(T) \neq \varnothing$. Let $f: C \rightarrow C$ be a fixed contractive mapping from $C$ to $C .\left\{x_{t}\right\}$ is defined by

$$
x_{t}=t f\left(x_{t}\right)+(1-t) P T x_{t},
$$

where $P$ is a sunny nonexpansive retraction of $E$ onto $C$, then $x_{t}$ converges strongly to some fixed point $q$ of $T$ as $t \rightarrow 0$ and $q$ is the unique solution in $F(T)$ to the following variational inequality:

$$
\langle(I-f) q, j(q-u)\rangle \quad \forall u \in F(T) .
$$

Lemma 3.3 ([6], Lemma 2.1). Let $\left\{\alpha_{n}\right\}$ be a sequence of nonnegative real numbers satisfying the property

$$
\alpha_{n+1} \leq\left(1-\gamma_{n}\right) \alpha_{n}+\delta_{n} \quad \forall n \geq 0,
$$

where $\left\{\gamma_{n}\right\} \in(0,1)$ and $\delta_{n}$ is a sequence in $\mathbb{R}$ such that:

(i) $\lim _{n \rightarrow \infty} \gamma_{n}=0$ and $\sum_{n=0}^{\infty} \gamma_{n}=\infty$;

(ii) either $\sum_{n=0}^{\infty} \delta_{n}<+\infty$ or $\lim \sup _{n \rightarrow \infty}\left(\delta_{n} / \gamma_{n}\right) \leq 0$,

then $\lim _{n \rightarrow \infty} \alpha_{n}=0$.

Theorem 3.4. Let E be a uniformly smooth Banach space, suppose that $C$ is a nonempty closed convex subset of $E, T: C \rightarrow E$ is a nonexpansive nonself-mapping satisfying the weakly inward condition, and $F(T) \neq \varnothing$. Let $f: C \rightarrow C$ be a fixed contractive mapping, and $\left\{x_{n}\right\}$ is defined by (1.5), where $P$ is a sunny nonexpansive retraction of $E$ onto $C$, and $\alpha_{n} \in(0,1)$ satisfies the following conditions:

(i) $\alpha_{n} \rightarrow 0$, as $n \rightarrow \infty$;

(ii) $\sum_{n=0}^{\infty} \alpha_{n}=\infty$;

(iii) either $\sum_{n=0}^{\infty}\left|\alpha_{n+1}-\alpha_{n}\right|<\infty$ or $\lim _{n \rightarrow \infty}\left(\alpha_{n+1} / \alpha_{n}\right)=1$.

Then $x_{n}$ converges strongly to a fixed point $q$ of $T$ such that $q$ is the unique solution in $F(T)$ to the following variational inequality:

$$
\langle(I-f) q, j(q-u)\rangle \leq 0 \quad \forall u \in F(T) .
$$

Proof. First we show $\left\{x_{n}\right\}$ is bounded. Take $u \in F(T)$, it follows that

$$
\begin{aligned}
\left\|x_{n+1}-u\right\| & =\left\|P\left(\left(1-\alpha_{n}\right) T x_{n}+\alpha_{n} f\left(x_{n}\right)\right)-P u\right\| \\
& \leq\left\|\left(1-\alpha_{n}\right) T x_{n}+\alpha_{n} f\left(x_{n}\right)-u\right\| \\
& \leq\left(1-\alpha_{n}\right)\left\|T x_{n}-u\right\|+\alpha_{n}\left(\left\|f\left(x_{n}\right)-f(u)\right\|+\|f(u)-u\|\right) \\
& \leq\left(1-\alpha_{n}\right)\left\|x_{n}-u\right\|+\alpha_{n}\left(\beta\left\|x_{n}-u\right\|+\|f(u)-u\|\right) \\
& =\left(1-(1-\beta) \alpha_{n}\right)\left\|x_{n}-u\right\|+\alpha_{n}\|f(u)-u\| \\
& \leq \max \left\{\left\|x_{n}-u\right\|, \frac{1}{1-\beta}\|f(u)-u\|\right\} .
\end{aligned}
$$


8 Strong convergence of approximation fixed points

By induction,

$$
\left\|x_{n}-u\right\| \leq \max \left\{\left\|x_{0}-u\right\|, \frac{1}{1-\beta}\|f(u)-u\|\right\}, \quad n \geq 0
$$

and $\left\{x_{n}\right\}$ is bounded, so are $\left\{T x_{n}\right\}$ and $\left\{f\left(x_{n}\right)\right\}$. We claim that

$$
x_{n+1}-x_{n} \longrightarrow 0 \quad \text { as } n \longrightarrow \infty \text {. }
$$

Indeed we have (for some appropriate constant $M>0$ )

$$
\begin{aligned}
\left\|x_{n+1}-x_{n}\right\|= & \left\|P\left(\alpha_{n} f\left(x_{n}\right)+\left(1-\alpha_{n}\right) T x_{n}\right)-P\left(\alpha_{n-1} f\left(x_{n-1}\right)+\left(1-\alpha_{n-1}\right) T x_{n-1}\right)\right\| \\
\leq & \left\|\alpha_{n} f\left(x_{n}\right)+\left(1-\alpha_{n}\right) T x_{n}-\alpha_{n-1} f\left(x_{n-1}\right)-\left(1-\alpha_{n-1}\right) T x_{n-1}\right\| \\
\leq & \left\|\left(1-\alpha_{n}\right)\left(T x_{n}-T x_{n-1}\right)+\left(\alpha_{n}-\alpha_{n-1}\right)\left(f\left(x_{n-1}\right)-T x_{n-1}\right)\right\| \\
& +\alpha_{n}\left\|f\left(x_{n}\right)-f\left(x_{n-1}\right)\right\| \\
\leq & \left(1-\alpha_{n}\right)\left\|x_{n}-x_{n-1}\right\|[3 p t]+M\left|\alpha_{n}-\alpha_{n-1}\right|+\beta \alpha_{n}\left\|x_{n}-x_{n-1}\right\| \\
= & \left(1-(1-\beta) \alpha_{n}\right)\left\|x_{n}-x_{n-1}\right\|[3 p t]+M\left|\alpha_{n}-\alpha_{n-1}\right| .
\end{aligned}
$$

By Lemma 3.3 we have $\left\|x_{n+1}-x_{n}\right\| \rightarrow 0$, as $n \rightarrow \infty$. We now show that

$$
\left\|x_{n}-P T x_{n}\right\| \longrightarrow 0
$$

In fact,

$$
\begin{aligned}
\left\|x_{n+1}-P T x_{n}\right\| & =\left\|P\left(\alpha_{n} f\left(x_{n}\right)+\left(1-\alpha_{n}\right) T x_{n}\right)-P T x_{n}\right\| \\
& \leq \alpha_{n}\left\|f\left(x_{n}\right)-T x_{n}\right\| .
\end{aligned}
$$

This follows from (3.30) that

$$
\begin{aligned}
\left\|x_{n}-P T x_{n}\right\| & \leq\left\|x_{n}-x_{n+1}\right\|+\left\|x_{n+1}-P T x_{n}\right\| \\
& \leq\left\|x_{n}-x_{n+1}\right\|+\alpha_{n}\left\|f\left(x_{n}\right)-T x_{n}\right\| \longrightarrow 0 \quad \text { as } n \longrightarrow \infty
\end{aligned}
$$

Let $q=\lim _{t \rightarrow 0} x_{t}$, where $\left\{x_{t}\right\}$ is defined in Corollary 3.2, we get that $q$ is the unique solution in $F(T)$ to the following variational inequality:

$$
\langle(I-f) q, j(q-u)\rangle \leq 0 \quad \forall u \in F(T)
$$

We next show that

$$
\limsup _{n \rightarrow \infty}\left\langle f(q)-q, j\left(x_{n}-q\right)\right\rangle \leq 0
$$


Form Corollary 3.2, let $x_{t}=t f\left(x_{t}\right)+(1-t) P T x_{t}$, indeed we can write

$$
x_{t}-x_{n}=t\left(f\left(x_{t}\right)-x_{n}\right)+(1-t)\left(P T x_{t}-x_{n}\right) .
$$

Noting (3.32), putting

$$
a_{n}(t)=\left\|x_{n}-P T x_{n}\right\|\left(\left\|x_{n}-P T x_{n}\right\|+2\left\|x_{n}-x_{t}\right\|\right) \longrightarrow 0 \quad \text { as } n \longrightarrow \infty,
$$

and using (2.2), we obtain

$$
\begin{aligned}
\| x_{t}- & x_{n} \|^{2} \\
\leq & (1-t)^{2}\left\|P T x_{t}-x_{n}\right\|^{2}+2 t\left\langle f\left(x_{t}\right)-x_{n}, j\left(x_{t}-x_{n}\right)\right\rangle \\
\leq & (1-t)^{2}\left\|P T x_{t}-P T x_{n}+P T x_{n}-x_{n}\right\|^{2}+2 t\left\langle f\left(x_{t}\right)-x_{t}, j\left(x_{t}-x_{n}\right)\right\rangle \\
& +2 t\left\|x_{t}-x_{n}\right\|^{2} \leq(1-t)^{2}\left\|x_{t}-x_{n}\right\|^{2}+(1-t)^{2}\left\|x_{n}-P T x_{n}\right\|^{2} \\
& +2(1-t)^{2}\left\|P T x_{n}-x_{n}\right\|\left\|x_{t}-x_{n}\right\|+2 t\left\langle f\left(x_{t}\right)-x_{t}, j\left(x_{t}-x_{n}\right)\right\rangle+2 t\left\|x_{t}-x_{n}\right\|^{2} \\
\leq & \left(1+t^{2}\right)\left\|x_{t}-x_{n}\right\|^{2}+a_{n}(t)+2 t\left\langle f\left(x_{t}\right)-x_{t}, j\left(x_{t}-x_{n}\right)\right\rangle .
\end{aligned}
$$

The last inequality implies

$$
\left\langle f\left(x_{t}\right)-x_{t}, j\left(x_{n}-x_{t}\right)\right\rangle \leq \frac{t}{2}\left\|x_{t}-x_{n}\right\|^{2}+\frac{1}{2 t} a_{n}(t) .
$$

From $a_{n}(t) \rightarrow 0$ as $n \rightarrow \infty$ we get

$$
\limsup _{n \rightarrow \infty}\left\langle f\left(x_{t}\right)-x_{t}, j\left(x_{n}-x_{t}\right)\right\rangle \leq M \cdot \frac{t}{2},
$$

where $M>0$ is a constant such that $M \geq\left\|x_{t}-x_{n}\right\|^{2}$ for all $n \geq 0$ and $t \in(0,1)$. By letting $t \rightarrow 0$ in (3.41) we have

$$
\lim _{t \rightarrow 0} \limsup _{n \rightarrow \infty}\left\langle f\left(x_{t}\right)-x_{t}, j\left(x_{n}-x_{t}\right)\right\rangle \leq 0
$$

On the one hand, for all $\varepsilon>0, \exists \delta_{1}$ such that $t \in\left(0, \delta_{1}\right)$,

$$
\limsup _{n \rightarrow \infty}\left\langle f\left(x_{t}\right)-x_{t}, j\left(x_{n}-x_{t}\right)\right\rangle \leq \frac{\varepsilon}{2}
$$


On the other hand, $\left\{x_{t}\right\}$ strongly converges to $q$, as $t \rightarrow 0$, the set $\left\{x_{t}-x_{n}\right\}$ is bounded, and the duality map $J$ is norm-to-norm uniformly continuous on bounded sets of uniformly smooth space $E$; from $x_{t} \rightarrow q(t \rightarrow 0)$, we get

$$
\begin{aligned}
& \left\|f(q)-q-\left(f\left(x_{t}\right)-x_{t}\right)\right\| \longrightarrow 0, \quad t \rightarrow 0, \\
& \left\|\left\langle f(q)-q, j\left(x_{n}-q\right)\right\rangle-\left\langle f\left(x_{t}\right)-x_{t}, j\left(x_{n}-x_{t}\right)\right\rangle\right\| \\
& =\left\|\left\langle f(q)-q, j\left(x_{n}-q\right)-j\left(x_{n}-x_{t}\right)\right\rangle+\left\langle f(q)-q-\left(f\left(x_{t}\right)-x_{t}\right), j\left(x_{n}-x_{t}\right)\right\rangle\right\| \\
& \leq\|f(q)-q\|\left\|j\left(x_{n}-q\right)-j\left(x_{n}-x_{t}\right)\right\| \\
& +\left\|f(q)-q-\left(f\left(x_{t}\right)-x_{t}\right)\right\|\left\|x_{n}-x_{t}\right\| \longrightarrow 0, \quad t \rightarrow 0 .
\end{aligned}
$$

Hence for the above $\varepsilon>0, \exists \delta_{2}$, such that for all $t \in\left(0, \delta_{2}\right)$, for all $n$, we have

$$
\left\|\left\langle f(q)-q, j\left(x_{n}-q\right)\right\rangle-\left\langle f\left(x_{t}\right)-x_{t}, j\left(x_{n}-x_{t}\right)\right\rangle\right\| \leq \frac{\varepsilon}{2} .
$$

Therefore, we have

$$
\left\langle f(q)-q, j\left(x_{n}-q\right)\right\rangle \leq\left\langle f\left(x_{t}\right)-x_{t}, j\left(x_{n}-x_{t}\right)\right\rangle+\frac{\varepsilon}{2} .
$$

Noting $\left(^{* *}\right)$ and taking $\delta=\min \left\{\delta_{1}, \delta_{2}\right\}$, for all $t \in(0, \delta)$, we have

$$
\begin{aligned}
& \limsup _{n \rightarrow \infty}\left\langle f(q)-q, j\left(x_{n}-q\right)\right\rangle \\
& \quad \leq \limsup _{n \rightarrow \infty}\left(\left\langle f\left(x_{t}\right)-x_{t}, j\left(x_{n}-x_{t}\right)\right\rangle+\frac{\varepsilon}{2}\right) \leq \frac{\varepsilon}{2}+\frac{\varepsilon}{2}=\varepsilon .
\end{aligned}
$$

Since $\varepsilon$ is arbitrary, we get

$$
\limsup _{n \rightarrow \infty}\left\langle f(q)-q, j\left(x_{n}-q\right)\right\rangle \leq 0 \text {. }
$$

Finally we show $x_{n} \rightarrow q$. Indeed

$$
x_{n+1}-\left(\alpha_{n} f\left(x_{n}\right)+\left(1-\alpha_{n}\right) q\right)=\left(x_{n+1}-q\right)-\alpha_{n}\left(f\left(x_{n}\right)-q\right) .
$$


By (2.2) we have

$$
\begin{aligned}
\left\|x_{n+1}-q\right\|^{2}= & \left\|x_{n+1}-\left(\alpha_{n} f\left(x_{n}\right)+\left(1-\alpha_{n}\right) q\right)+\alpha_{n}\left(f\left(x_{n}\right)-q\right)\right\|^{2} \\
\leq & \left\|x_{n+1}-P\left(\alpha_{n} f\left(x_{n}\right)+\left(1-\alpha_{n}\right) q\right)\right\|^{2}+2 \alpha_{n}\left\langle f\left(x_{n}\right)-q, j\left(x_{n+1}-q\right)\right\rangle \\
\leq & \left\|P\left[\alpha_{n} f\left(x_{n}\right)+\left(1-\alpha_{n}\right) T x_{n}\right]-P\left(\alpha_{n} f\left(x_{n}\right)+\left(1-\alpha_{n}\right) q\right)\right\|^{2} \\
& +2 \alpha_{n}\left\langle f\left(x_{n}\right)-q, j\left(x_{n+1}-q\right)\right\rangle \\
\leq & \left(1-\alpha_{n}\right)^{2}\left\|T x_{n}-q\right\|^{2}+2 \alpha_{n}\left\langle f\left(x_{n}\right)-f(q), j\left(x_{n+1}-q\right)\right\rangle \\
& +2 \alpha_{n}\left\langle f(q)-q, j\left(x_{n+1}-q\right)\right\rangle \\
\leq & \left(1-\alpha_{n}\right)^{2}\left\|x_{n}-q\right\|^{2}+2 \alpha_{n}\left\|f(q)-f\left(x_{n}\right)\right\|\left\|x_{n+1}-q\right\| \\
& +2 \alpha_{n}\left\langle f(q)-q, j\left(x_{n+1}-q\right)\right\rangle \\
\leq & \left(1-\alpha_{n}\right)^{2}\left\|x_{n}-q\right\|^{2}+\alpha_{n}\left(\left\|f(q)-f\left(x_{n}\right)\right\|^{2}+\left\|x_{n+1}-q\right\|^{2}\right) \\
& +2 \alpha_{n}\left\langle f(q)-q, j\left(x_{n+1}-q\right)\right\rangle .
\end{aligned}
$$

Therefore, we have

$$
\begin{aligned}
& \left(1-\alpha_{n}\right)\left\|x_{n+1}-q\right\|^{2} \\
& \quad \leq\left(1-\alpha_{n}\right)^{2}\left\|x_{n}-q\right\|^{2}+\alpha_{n} \beta^{2}\left\|x_{n}-q\right\|^{2}+2 \alpha_{n}\left\langle f(q)-q, j\left(x_{n+1}-q\right)\right\rangle .
\end{aligned}
$$

That is,

$$
\begin{aligned}
\left\|x_{n+1}-q\right\|^{2} \leq & \left(1-\frac{1-\beta^{2}}{1-\alpha_{n}} \alpha_{n}\right)\left\|x_{n}-q\right\|+\frac{\alpha_{n}^{2}}{1-\alpha_{n}}\left\|x_{n}-q\right\|^{2} \\
& +\frac{2 \alpha_{n}}{1-\alpha_{n}}\left\langle f(q)-q, j\left(x_{n+1}-q\right)\right\rangle \\
\leq & \left(1-\gamma_{n}\right)\left\|x_{n}-q\right\|^{2}+\lambda \gamma_{n} \alpha_{n}+\frac{2}{1-\beta^{2}} \gamma_{n}\left\langle f(q)-q, j\left(x_{n+1}-q\right)\right\rangle,
\end{aligned}
$$

where $\gamma_{n}=\left(\left(1-\beta^{2}\right) /\left(1-\alpha_{n}\right)\right) \alpha_{n}$ and $\lambda$ is a constant such that $\lambda>\left(1 /\left(1-\beta^{2}\right)\right)\left\|x_{n}-q\right\|^{2}$.

Hence,

$$
\left\|x_{n+1}-q\right\|^{2} \leq\left(1-\gamma_{n}\right)\left\|x_{n}-q\right\|^{2}+\gamma_{n}\left(\lambda \alpha_{n}+\frac{2}{1-\beta^{2}}\left\langle f(q)-q, j\left(x_{n+1}-q\right)\right\rangle\right) .
$$

It is easily seen that $\gamma_{n} \rightarrow 0, \sum_{n=1}^{\infty} \gamma_{n}=\infty$, and (noting (3.36))

$$
\limsup _{n \rightarrow \infty}\left(\lambda \alpha_{n}+\frac{2}{1-\beta^{2}}\left\langle f(q)-q, j\left(x_{n+1}-q\right)\right\rangle\right) \leq 0 .
$$

Applying Lemma 3.3 onto (3.52), we have $x_{n} \rightarrow q$.

The proof is complete. 


\section{Strong convergence of approximation fixed points}

\section{Acknowledgment}

This work is supported by the National Science Foundation of China, Grants 10471033 and 10271011.

\section{References}

[1] N. Shahzad, Approximating fixed points of non-self nonexpansive mappings in Banach spaces, Nonlinear Analysis 61 (2005), no. 6, 1031-1039.

[2] Y. Song and R. Chen, Viscosity approximation methods for nonexpansive nonself-mappings, Journal of Mathematical Analysis and Applications 321 (2006), no. 1, 316-326.

[3] W. Takahashi, Nonlinear Functional Analysis. Fixed Point Theory and Its Applications, Yokohama Publishers, Yokohama, 2000.

[4] W. Takahashi and G.-E. Kim, Strong convergence of approximants to fixed points of nonexpansive nonself-mappings in Banach spaces, Nonlinear Analysis. Theory, Methods \& Applications 32 (1998), no. 3, 447-454.

[5] H.-K. Xu, Approximating curves of nonexpansive nonself-mappings in Banach spaces, Comptes Rendus de l'Académie des Sciences. Série I. Mathématique 325 (1997), no. 2, 151-156.

[6] _ Viscosity approximation methods for nonexpansive mappings, Journal of Mathematical Analysis and Applications 298 (2004), no. 1, 279-291.

Rudong Chen: Department of Mathematics, Tianjin Polytechnic University,

Tianjin 300160, China

E-mail address: chenrd@tjpu.edu.cn

Zhichuan Zhu: Department of Mathematics, Tianjin Polytechnic University, Tianjin 300160, China

E-mail address: zhuzcnh@yahoo.com.cn 


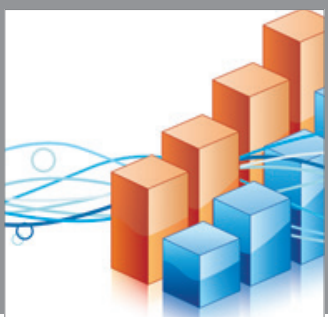

Advances in

Operations Research

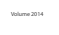

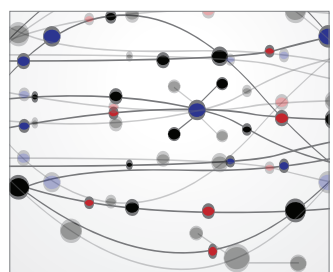

\section{The Scientific} World Journal
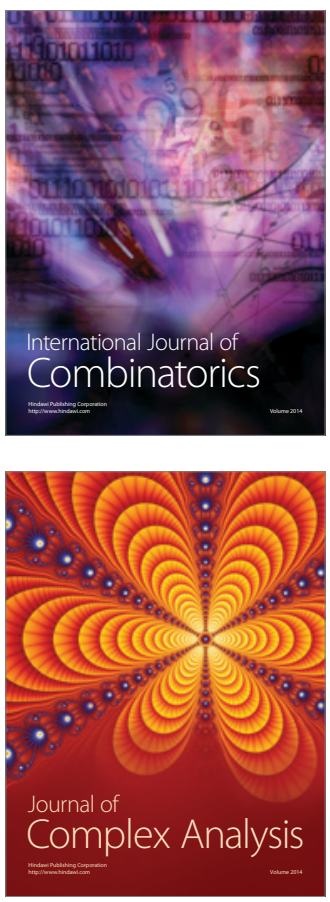

International Journal of

Mathematics and

Mathematical

Sciences
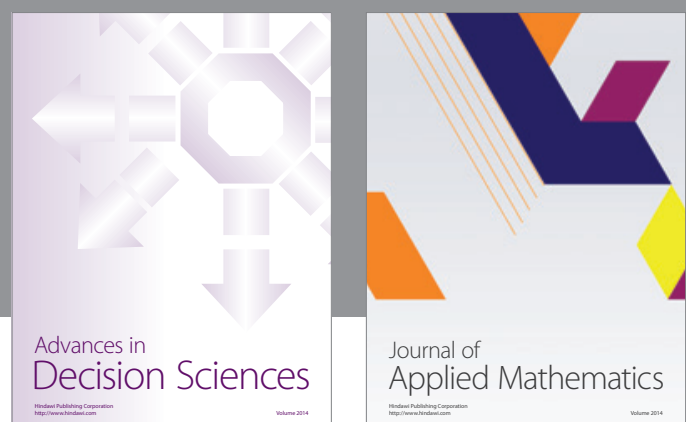

Journal of

Applied Mathematics
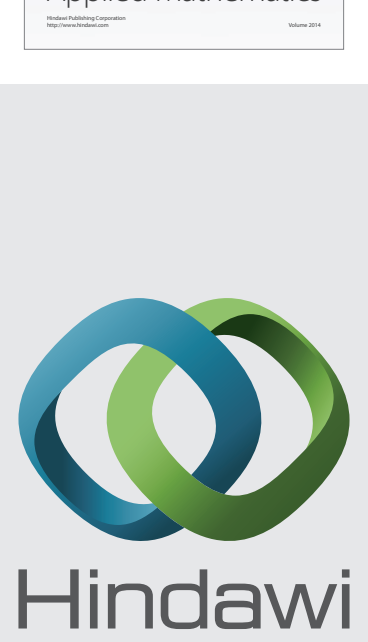

Submit your manuscripts at http://www.hindawi.com
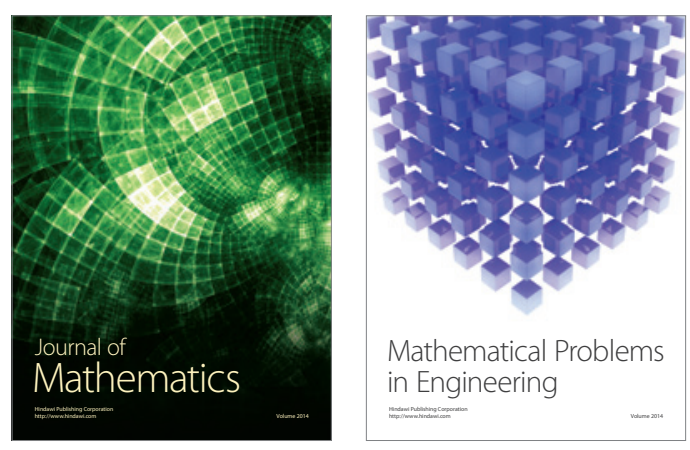

Mathematical Problems in Engineering
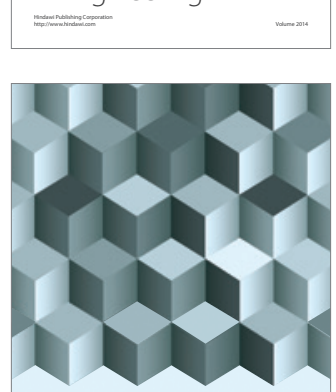

Journal of

Function Spaces
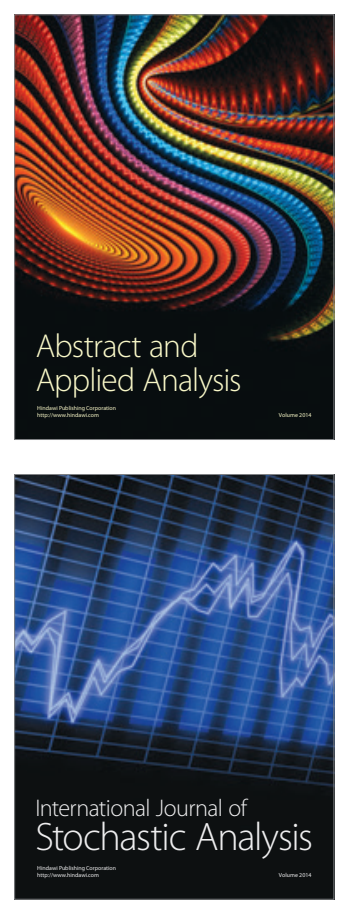

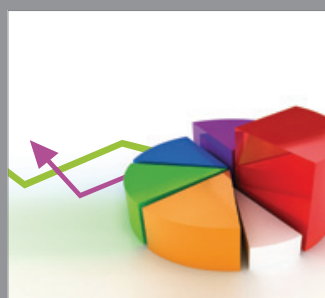

ournal of

Probability and Statistics

Promensencen
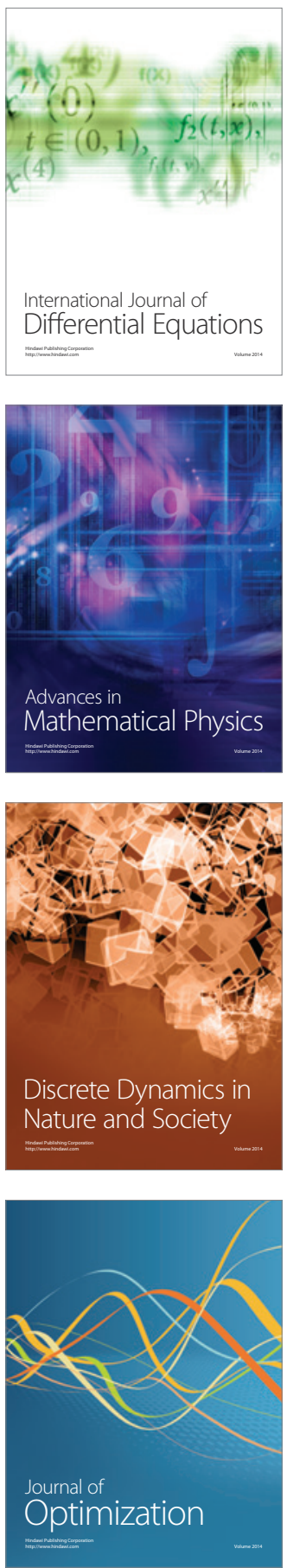